\title{
A novel and essential mechanism determining specificity and activity of protein phosphatase 2A (PP2A) in vivo
}

\author{
Thomas Fellner, ${ }^{1}$ Daniel H. Lackner, ${ }^{1}$ Hans Hombauer, ${ }^{1}$ Patrick Piribauer, Ingrid Mudrak, \\ Katrin Zaragoza, Claudia Juno, and Egon Ogris ${ }^{2}$
}

Institute of Medical Biochemistry, Division of Molecular Biology, Vienna Biocenter, University of Vienna, A-1030 Vienna, Austria

Protein phosphatase 2A (PP2A) is an essential intracellular serine/threonine phosphatase containing a catalytic subunit that possesses the potential to dephosphorylate promiscuously tyrosine-phosphorylated substrates in vitro. How PP2A acquires its intracellular specificity and activity for serine/threonine-phosphorylated substrates is unknown. Here we report a novel and phylogenetically conserved mechanism to generate active phospho-serine/threonine-specific PP2A in vivo. Phosphotyrosyl phosphatase activator (PTPA), a protein of so far unknown intracellular function, is required for the biogenesis of active and specific PP2A. Deletion of the yeast PTPA homologs generated a PP2A catalytic subunit with a conformation different from the wild-type enzyme, as indicated by its altered substrate specificity, reduced protein stability, and metal dependence. Complementation and RNA-interference experiments showed that PTPA fulfills an essential function conserved from yeast to man.

[Keywords: PP2A biogenesis; PTPA; metals; active site]

Received January 14, 2003; revised version accepted June 30, 2003.

Protein phosphorylation is a posttranslational modification, mostly reversible, that is used in cells for the regulation of multiple processes. Analyses of eukaryotic genomes reveal that the genes coding for protein kinases, the enzymes catalyzing the phosphorylation reaction, outnumber by two- to threefold genes for protein phosphatases, the enzymes catalyzing dephosphorylation (Zolnierowicz 2000). Protein phosphatases counterbalance the activity of the large number of substrate-specific kinases by the combinatorial assembly of holoenzymes with different substrate specificity. Holoenzymes of a certain protein phosphatase family consist of a common catalytic subunit associated with different regulatory subunits that determine substrate targeting and modulate catalytic activity. Hence, the catalytic subunits of the major protein phosphatase families are produced in abundance. For instance, the catalytic subunit (C subunit) of protein phosphatase $2 \mathrm{~A}$ (PP2A), comprises, dependent on the cell type, $0.3 \%-1 \%$ of total cellular protein (Virshup 2000).

Based on its specificity for phosphorylated serine/ threonine residues, the PP2A C subunit belongs to the family of eukaryotic protein-serine/threonine phospha-

\footnotetext{
${ }^{1}$ These authors contributed equally to this work.

${ }^{2}$ Corresponding author.

E-MAIL eo@mol.univie.ac.at; FAX 43-1-4277-9617.

Article and publication are at http://www.genesdev.org/cgi/doi/10.1101/ gad.259903.
}

tases (PSTPs). PSTPs possess a catalytic core structure that is distinct from the core of protein tyrosine phosphatases (PTPs) and dual specificity phosphatases (DSPs). In consequence of the structural differences, the different protein phosphatase families use distinct catalytic mechanisms for the hydrolysis of the phosphoester bond. In contrast to PTPs (and the DSP subfamily) PSTPs are metallo-phosphoesterases that require metals in the active site for catalysis and for their structural integrity. When isolated from eukaryotic sources, the PSTP family members protein phosphatase 1 (PP1) and PP2A ("native" PP1 or PP2A) do not require the addition of metal ions for their activity. However, PP1 and PP2A convert into metal-dependent enzymes during long-term storage or on treatment with the phosphatase inhibitors ATP, pyrophosphate (PPi), or NaF (Burchell and Cohen 1978; Hsiao et al. 1978; Cai et al. 1995). In addition, bacterially expressed PP1 has biochemical properties very similar to the long-term stored or ATP/PPi-inhibited native enzyme. Attempts to express soluble and catalytically active PP2A in bacteria were unsuccessful so far, and consequently no crystal structure of the PP2A catalytic subunit exists. The metal-dependent forms of PP1 and PP2A possess broadened substrate specificity shown by the ability to dephosphorylate phospho-tyrosine (P-Tyr)-containing substrates and para-nitrophenyl phosphate (pNPP; Goris et al. 1988; Alessi et al. 1993). The transient activation of PP2A's phosphotyrosyl activity can be further increased in vitro by a highly conserved protein 
termed phosphotyrosyl phosphatase activator (PTPA; Cayla et al. 1990). However, it is not known whether PP2A ever functions as a phosphotyrosyl phosphatase in vivo, and thus the physiological role of PTPA is so far unclear.

More recently Rempola et al. (2000) conducted a functional analysis of novel yeast genes corresponding to the open reading frames (ORFs) YIL153w and YPL152w, which code for proteins that are $38 \%$ and $37 \%$ identical, respectively, to human PTPA. They found that deletion of the ORFs causes, among other phenotypes, rapamycin resistance, and thus these two genes were termed $R R D 1$ and $R R D 2$ for rapamycin resistant deletion (Rempola et al. 2000). Rapamycin resistance is also observed in strains lacking genes for the regulatory subunits of PP2A or for strains carrying certain alleles of TAP42. TAP42 interacts with the catalytic subunit of PP2A as well as with the PP2A-related phosphatase SIT4 (Di Como and Arndt 1996) and seems to act as an inhibitor of these phosphatases in the target of rapamycin (TOR) signaling pathway (Beck and Hall 1999; Jiang and Broach 1999). The common phenotype of rapamycin resistance pointed to a potential functional relation between the RRD proteins and the PP2A/SIT4 phosphatases. Indeed, genetic and biochemical evidence suggests that SIT4 is an intracellular target of RRD1 and potentially also of RRD2 (Mitchell and Sprague 2001). Deletion of both RRD genes is synthetically lethal, whereas the SIT4 deletion strain is viable, indicating that the RRD proteins must have targets other than SIT4. One target of RRD2 may be yeast PP2A, because synthetic lethality of the double $R R D$ deletion strain can be suppressed by the overexpression of the yeast PP2A C subunit isoform PPH22 (Rempola et al. 2000). The pleiotropic phenotypes of the single $R R D$ deletion strains such as aberrant bud morphology, altered proliferation rate, and a defective spindle checkpoint indicated that a basic function of PP2A and PP2A-related phosphatases might be affected (Van Hoof et al. 2001). What this basic function could be remained unresolved.

We present evidence that RRD1 and RRD2 are required for generation of active and phospho-serine/threonine (P-Ser/P-Thr)-specific PP2A and SIT4 in vivo. Loss of the RRD proteins resulted in generation of a PP2A catalytic subunit, which differs from the wild-type enzyme in terms of substrate specificity for P-Ser/P-Thr over P-Tyr, protein stability, and metal dependence. The altered biochemical properties suggest that the catalytic subunit produced in the $r r d 1 \Delta / r r d 2 \Delta$ strain is conformationally different from PP2A in a wild-type strain. However, the structural alterations caused by the absence of RRD functions did not hamper PP2A complex assembly, as shown by the ability of the catalytic $\mathrm{C}$ subunit to interact with the regulatory A and B subunits. Thus, RRD functions are not required for holoenzyme assembly with the A and B subunits, but rather for the formation of an active PP2A enzyme with correct substrate specificity for P-Ser/P-Thr over P-Tyr. Complementation experiments showed that the generation of active and specific PP2A by RRD is a novel and fundamental mechanism conserved from yeast to man. Moreover, RRD/PTPA function is essential for cell survival because suppression of PTPA expression by RNAi triggered apoptotic cell death.

\section{Results}

A PP2A active site mutant forms a stable complex with PTPA, the mammalian homolog of the yeast RRD proteins

Individual substitutions of absolutely conserved amino acids within the active site of the PP2A catalytic subunit abolish its catalytic activity (Ogris et al. 1999b). Several of these mutants interact stably with cellular proteins that bind only transiently to wild-type C subunit (Ogris et al. 1999a). We identified one of them as a protein phosphatase methylesterase-1 (PME-1), an enzyme that is able to demethylate the PP2A C subunit in vitro. Several additional cellular proteins associated with PP2A activesite mutants. Immunoprecipitates of hemagglutinin (HA)-tagged inactive mutant H59S from lysates of ${ }^{35} \mathrm{~S}$ labeled cells stably expressing this $C$ subunit mutant were analyzed on two-dimensional gels (Fig. 1A). Two cellular proteins of estimated molecular weights of 37 and $39 \mathrm{kD}$ and isoelectric points of 6.2 and 6.0, respectively, coimmunoprecipitated with the mutant but not wild-type C subunit or vector control. HA-tagged H59S complexes were purified by anti-tag immunoaffinity column and preparative two-dimensional gel electrophoresis and the coprecipitated $37-\mathrm{kD}$ protein was identified by peptide mapping and sequencing as PTPA. PTPA is known for its ability to convert, in an ATP $/ \mathrm{Mg}^{2+}$-dependent reaction, the serine/threonine phosphatase PP2A into a tyrosine phosphatase in vitro (Cayla et al. 1990). However, only a weak interaction between wild-type PP2A and PTPA could be detected by PTPA-affinity chromatography (Van Hoof et al. 1994).

To confirm the interaction between the inactive $\mathrm{C}$ subunit mutant H59S and PTPA, we carried out reciprocal immunoprecipitations with the tagged proteins (Fig. 1B,C). We generated NIH3T3 fibroblasts stably expressing HA-tagged wild-type or mutant $\mathrm{C}$ subunit, alone or together with myc-tagged wild-type PTPA. From lysates of these cell lines we immunoprecipitated HA-tagged C subunits or myc-tagged PTPA and analyzed the immunoprecipitates for the presence of the respective tagged protein as well as for the presence of the endogenous protein (Fig. 1B,C). The inactive C subunit mutant H59S associated with substantial amounts of endogenous PTPA as well as ectopically expressed myc-tagged PTPA (Fig. 1B, bottom panel). In contrast, only small amounts of the overexpressed myc-tagged PTPA could be detected in wild-type $C$ subunit immunoprecipitates (Fig. 1B, lane 3), confirming a weak interaction between PP2A and PTPA (Van Hoof et al. 1994). Immunoprecipitation via myc-tagged PTPA showed again that the inactive mutant H59S bound with high affinity to PTPA (Fig. 1C, lane 4), whereas wild-type C subunit (untagged or HAtagged) interacted only weakly with PTPA (Fig. 1C, lanes 3-5). 

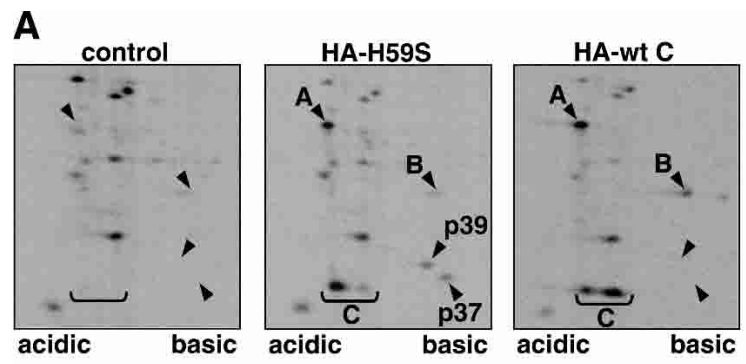

B

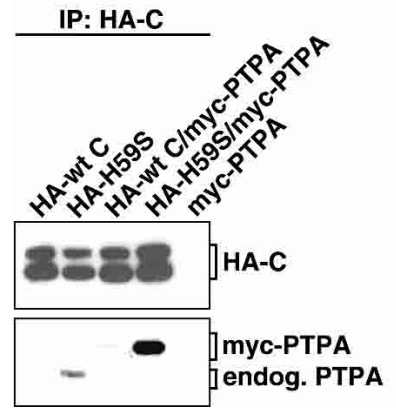

C
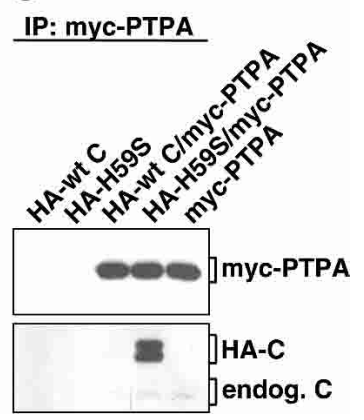

Figure 1. A PP2A active site mutant forms a stable complex with PTPA, the mammalian homolog of the yeast RRD proteins. (A) Anti-HA tag immunoprecipitates from lysates of ${ }^{35} \mathrm{~S}$ methionine-labeled NIH3T3 cells stably expressing HA-tagged wild-type $\mathrm{C}$ subunit (HA-wt C), or the HA-tagged mutant H59S (HA-H59S), or containing empty pBabe-puro vector (control) were analyzed by two-dimensional gel electrophoresis and autoradiography. The portion of each gel containing immunopurified HA-tagged C subunit (bracket labeled C), PP2A A subunit (arrowhead labeled A), PP2A B subunit (arrowhead labeled B), PTPA (arrowhead labeled p37), and the protein p39 (arrowhead labeled p39) is shown. Unlabeled arrowheads and brackets indicate the corresponding positions of the immunoprecipitated proteins in the control and HA-wt C panels. $(B, C)$ Anti-HA tag $(B)$ or anti-myc tag $(C)$ immunoprecipitates from lysates of NIH3T3 cells stably expressing HA-wt C, HA-H59S, or myctagged PTPA and from lysates of cells coexpressing myc-tagged PTPA and HA-wt C or HA-H59S were analyzed by $10 \%$ SDSPAGE and immunoblotting either with anti-C subunit to detect both HA-tagged (HA-C) and endogenous PP2A C subunit (endog. C) or anti-PTPA antibody to visualize both myc-tagged (myc-PTPA) and endogenous PTPA (endog. PTPA).

\section{Mammalian PTPA can complement a mutant of the yeast PTPA genes RRD1 and RRD2}

To elucidate the in vivo function of PTPA, we undertook a genetic and biochemical analysis in Saccharomyces cerevisiae. Recent work from several groups showed that deletion of both yeast $R R D$ genes is synthetically lethal, but can be rescued through an unknown mechanism by expression of the viable allele of the polymorphic gene SSD1 (SSD1-V; Rempola et al. 2000). We generated a second double deletion strain, $\operatorname{rrd} 1 \Delta / r r d 2 \Delta$, which was not lethal, most likely due to the genetic background of the strain, but which showed a severe proliferation defect at $16^{\circ} \mathrm{C}$ and a lesser defect at $37^{\circ} \mathrm{C}$ and $30^{\circ} \mathrm{C}$ (Fig. 2A). The single disruption strains grew slightly slower than the wild-type strain, with the most pronounced effect seen for $\operatorname{rrd} 1 \Delta$ at $16^{\circ} \mathrm{C}$. The observed proliferation phenotype of the $r r d 1 \Delta / r r d 2 \Delta$ strain indicated that an important intracellular function must have been affected by the disruption of these genes.

To test whether expression of mammalian PTPA is able to functionally replace the yeast homologs, we expressed myc-tagged mouse PTPA in the wild-type and the $\operatorname{rrd} 1 \Delta / r r d 2 \Delta$ strain, and we analyzed proliferation of the transformed strains at different temperatures (Fig. 2B). The $r r d 1 \Delta / r r d 2 \Delta$ strain expressing mammalian PTPA proliferated almost as well as the wild-type strain at the three tested temperatures, indicating the conservation of functions between mammalian and yeast PTPA. The partial complementation of the proliferation phenotype probably reflects the fact that mammalian PTPA was expressed at very low levels, even under the control of the strong TPI promoter (data not shown).

\section{Suppression of PTPA expression by RNAi triggers apoptotic cell death in mammalian cells}

Deletion of the yeast PTPA homologs caused a severe proliferation defect in the BY strain or synthetic lethality in strains with a different genetic background. To determine the importance of PTPA function in mammalian cells, we expressed short interfering RNA (siRNA) targeted against the PTPA coding sequence in HeLa cells. Cells were transfected with the pSUPER vector containing a 19-bp double-stranded PTPA sequence (PTPARNAi) or a nonsense oligonucleotide (NS-RNAi) or the vector alone (pSUPER; Brummelkamp et al. 2002). For detection and selection of transfected cells, a vector coding for the expression of green fluorescent protein (GFP) and the puromycin resistance gene was cotransfected with the pSUPER constructs. Less than $5 \%$ of wild-type

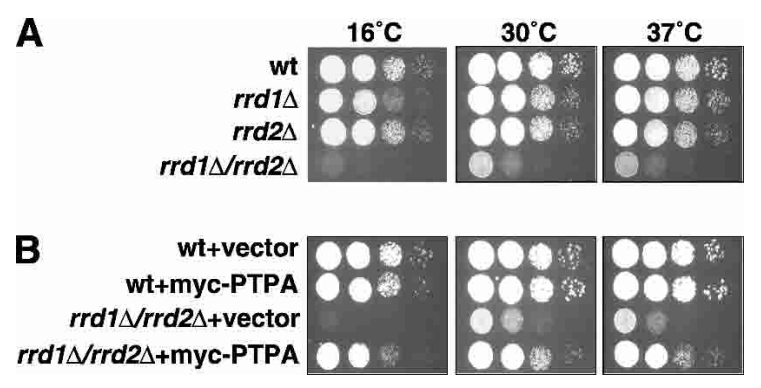

Figure 2. Mammalian PTPA can complement a mutant of the yeast PTPA genes $R R D 1$ and $R R D 2$. (A) Exponentially growing cultures of the wild-type strain BY4741 (wt) and the strains deleted for RRD1 (rrd1 $\Delta), R R D 2$ (rrd2 $)$, or both RRD1 and $R R D 2(r r d 1 \Delta / r r d 2 \Delta)$ were 10-fold serially diluted in YPD liquid medium, spotted on YPD plates, and incubated at $16^{\circ} \mathrm{C}, 30^{\circ} \mathrm{C}$, and $37^{\circ} \mathrm{C}$. Growth is shown after incubation at $30^{\circ} \mathrm{C}$ and $37^{\circ} \mathrm{C}$ for $2 \mathrm{~d}$ or after $7 \mathrm{~d}$ at $16^{\circ} \mathrm{C}$. (B) Exponentially growing cultures of the wild-type and the $r r d 1 \Delta / r r d 2 \Delta$ strain, containing either empty vector (vector) or expressing myc-tagged mammalian PTPA (myc-PTPA) were 10-fold serially diluted in CM-Leu liquid medium supplemented with glucose and spotted on glucosecontaining CM-Leu plates. Plates were incubated at $30^{\circ} \mathrm{C}$ and $37^{\circ} \mathrm{C}$ for $3 \mathrm{~d}$ and at $16^{\circ} \mathrm{C}$ for $9 \mathrm{~d}$. 
A

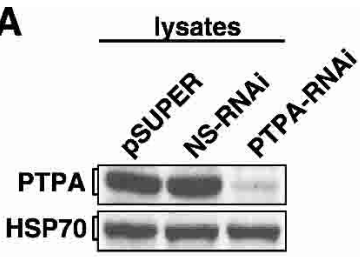

B
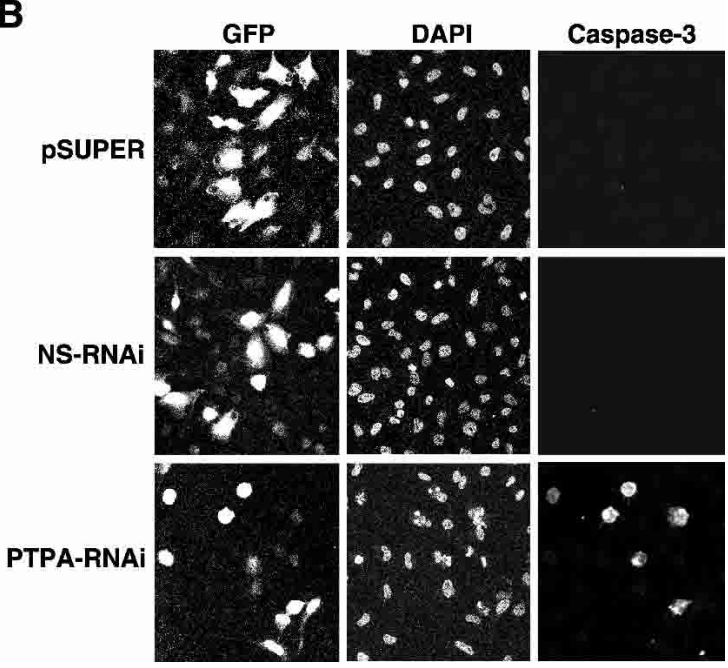

Figure 3. Suppression of PTPA expression by RNAi triggers apoptotic cell death in mammalian cells. (A) HeLa cells were cotransfected with the pRETRO $_{\text {Katze }}$ vector containing the coding sequence of the puromycin resistance marker and of the green fluorescence protein (GFP) and either empty pSUPER vector (pSUPER), pSUPER-NS-PTPA containing a "nonsense"-targeting sequence (NS-RNAi), or pSUPER-PTPA containing a PTPA-targeting sequence (PTPA-RNAi). After transfection, cells were grown in medium supplemented with puromycin for $48 \mathrm{~h}$. Whole-cell lysates were analyzed by $10 \%$ SDS-PAGE and immunoblotting with anti-PTPA and anti-HSP70 (loading control) antibody. (B) HeLa cells were cotransfected as described in $A$, and $48 \mathrm{~h}$ after transfection, cells were stained with antibody specific for active caspase- 3 and counterstained with DAPI. GFP staining indicates cotransfected cells. Confocal microscopy images of representative cells are shown.

PTPA levels could be detected in lysates of HeLa cells 4 d posttransfection of the PTPA-RNAi construct, whereas PTPA levels were unchanged in NS-RNAi or pSUPER transfected cells (Fig. 3A). To determine the effects of PTPA down-regulation on cell survival in mammalian cells, we labeled transfected cells with an antibody specific for active caspase- 3 and counterstained them with DAPI (Fig. 3B). In contrast to the vector-transfected control cells, PTPA-RNAi transfected cells underwent apoptotic cell death, indicating that PTPA function is essential for cell survival in mammalian cells.

The heterotrimeric PP2A complex assembles correctly but is catalytically altered in the absence of RRD/PTPA functions

The phenotypes of the $r r d \Delta$ strains, namely, rapamycin resistance and a defective spindle assembly checkpoint
(Van Hoof et al. 2001), resemble the phenotypes of strains defective in PP2A complex assembly (Wu et al. 2000; Wei et al. 2001). We therefore analyzed PP2A complex assembly in isogenic wild type, $r r d 1 \Delta, \operatorname{rrd} 2 \Delta, p G A L$ $R R D 1 / r r d 2 \Delta$, and ppm $1 \Delta$ strains expressing HA-tagged yeast PP2A catalytic subunit PPH21. In the early stages of our study we used a strain termed $p G A L-R R D 1 / r r d 2 \Delta$, in which the expression of RRD1 is under control of the GAL1 promoter and thus expression of RRD1 could be turned off in glucose-containing medium. This strain was used in the experiments shown in Figure 4A and B, as described in Materials and Methods. Side-by-side comparison between this strain and the $\operatorname{rrd} 1 \Delta / \mathrm{rrd} 2 \Delta$ strain showed no differences in regard to the examined properties (data not shown). Anti-HA tag immunoprecipitates from lysates of these strains were analyzed for the presence of PP2A regulatory subunit A (TPD3) and B (CDC55) by SDS-PAGE and immunoblotting. PP2A complex assembly with the A and B subunit was not impaired in any of the $r r d \Delta$ strains (Fig. 4A), whereas deletion of the PP2A methyltransferase gene PPM-1 led to decreased TPD3 and CDC55 binding, as reported previously (Wu et al. 2000; Wei et al. 2001). CDC55 in complexes from wild-type and mutant strains migrated as a doublet in SDS-PAGE. However, the amount of shifted CDC55 was increased in PP2A complexes isolated from the $p G A L-R R D 1 / r r d 2 \Delta$ strain (Fig. 4A lower panel, lane 5). Densitometric quantitation of the upper and lower bands (from three independent experiments) revealed a change in the ratio of upper to lower band from $1 / 3.7$ in the wild-type strain to $1 / 1.4$ in the $p G A L-R D D 1 /$ rrd2s strain. Whether phosphorylation or a different posttranslational modification caused the shift will be the subject of future studies.

Because complex formation with the regulatory A and B subunits was unaffected, we analyzed the catalytic activity of HA-tagged $\mathrm{PPH} 21$ immunoprecipitates from lysates of the wild-type and deletion strains toward two different P-serine/P-threonine-phosphorylated substrates (Fig. 4B). Histone $\mathrm{H} 1$ phosphorylated by cdc2 kinase has been shown to be specifically dephosphorylated by heterotrimeric complexes containing the B subunit (Sola et al. 1991), whereas phosphorylase a is a general substrate for mono-, di-, and trimeric PP2A complexes (Cohen 1989). With either substrate, PP2A complexes isolated from the $\operatorname{rrd} 1 \Delta$ strain were only slightly less active than the wild-type enzyme, PP2A from the $\operatorname{rrd} 2 \Delta$ strain showed a $45 \%-50 \%$ reduction of activity, and PP2A from the $p G A L-R R D 1 / r r d 2 \Delta$ strain showed a substantial $80 \%-85 \%$ decrease (Fig. 4B). A similar reduction in PP2A catalytic activity was detected with other serine/ threonine-phosphorylated substrates like RCM-lysozyme phosphorylated by cAMP-dependent protein kinase or two phosphopeptide substrates LRRApSVA and KRpTIRR (data not shown). Deletion of the PP2A methyltransferase caused a marked decrease of the activity toward the B subunit specific substrate, cdc2-phosphorylated histone $\mathrm{H} 1$. This result is in line with recent findings showing that loss of PP2A methylation correlates with loss of B subunit binding (Wu et al. 2000; Wei et al. 

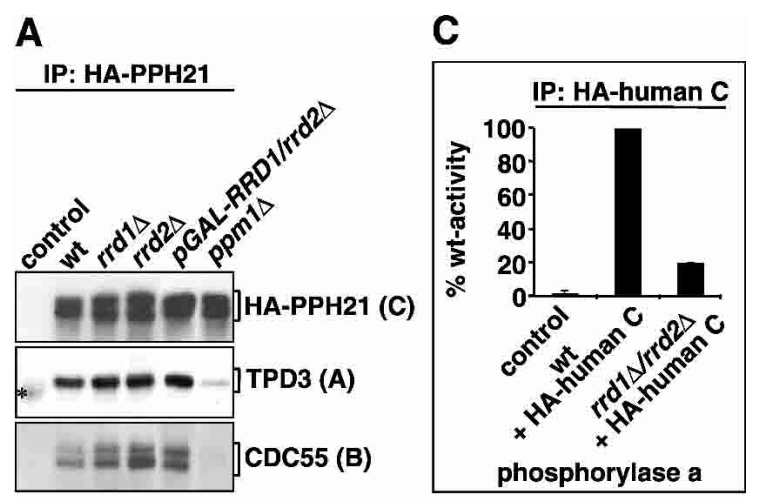

B

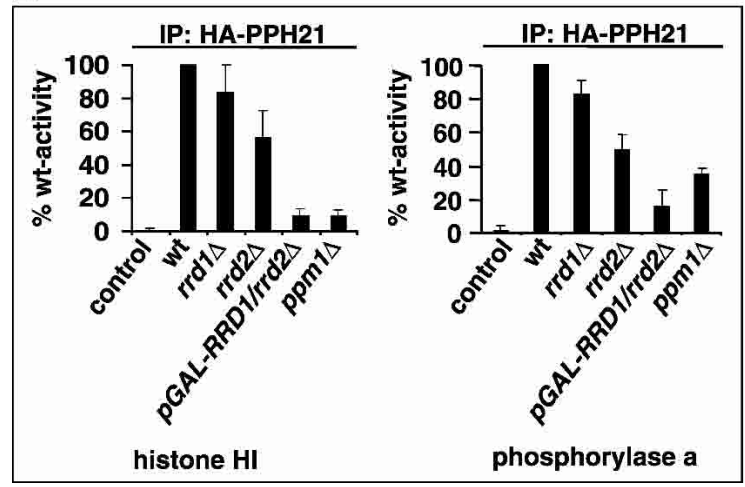

Figure 4. The heterotrimeric PP2A complex assembles correctly but is catalytically altered in the absence of RRD/PTPA functions. (A) Anti-HA tag immunoprecipitates from lysates of wild-type cells expressing untagged PPH21 (control) and of wildtype, $\operatorname{rrd1\Delta }, \operatorname{rrd} 2 \Delta, p p m 1 \Delta$, and $p G A L-R R D 1 / \mathrm{rrd2 \Delta}$ cells expressing HA-tagged PPH21 were analyzed by $7.5 \%$ SDS-PAGE and immunoblotting. Blots were sequentially incubated with anti-TPD3, anti-CDC55, and anti-HA antibody to visualize the yeast A, B, and HA-tagged C subunit of PP2A. The asterisk indicates a background band visible in lane 1 (control). Lanes 4-6 were not adjacent on the original blot. (B) Anti-HA tag immunoprecipitates from lysates of strains described in $A$ were analyzed by phosphatase assays toward the substrates cdc2 phosphorylated histone $\mathrm{HI}$ and phosphorylase a (see Materials and Methods for details). (C) Anti-HA tag immunoprecipitates from lysates of wild-type cells containing empty vector (control) and of wild-type and $r r d 1 \Delta / r r d 2 \Delta$ cells expressing HA-tagged human $\mathrm{C}$ subunit were assayed for catalytic activity toward phosphorylase a (see Materials and Methods for details).

2001). The $60 \%$ decrease of catalytic activity toward phosphorylase a was unexpected because the amount of free monomeric C subunit was increased in the ppm1s strain. Free C subunit, however, is known to possess in vitro a higher activity than PP2A dimers or trimers toward this substrate (Cohen 1989). Thus, loss of C subunit methylation in vivo correlated with a decrease of catalytic activity in general, independent of the decreased holoenzyme formation.

To determine whether RRD proteins are required for the activation of a mammalian $\mathrm{C}$ subunit, we transformed wild-type or $\operatorname{rrd} 1 \Delta / \mathrm{rrd} 2 \Delta$ strains with an expres- sion vector coding for HA-tagged human $\mathrm{C}$ subunit, and we tested immunoprecipitates of HA-tagged $\mathrm{C}$ for catalytic activity toward phosphorylase a (Fig. 4C). The activity of human $\mathrm{C}$ subunit in the $r r d 1 \Delta / r r d 2 \Delta$ strain was severely diminished, very similar to what we observed with the yeast $C$ subunit. These data indicated that the RRD proteins are required for the generation of active and specific PP2A and that the mechanism is conserved from yeast to man.

\section{The functional interaction between RRD/PTPA and PP2A is evolutionarily conserved}

To determine whether mammalian PTPA could functionally replace RRD proteins in activating PPH21 catalytic activity, we coexpressed HA-tagged PPH21 and myc-tagged mammalian PTPA in the wild-type or $r r d 1 \Delta /$ $\operatorname{rrd2} \Delta$ strain and measured the catalytic activities of HAPPH21 immunoprecipitates toward phosphorylase a (Fig. 5A) and cdc2-phosphorylated histone $\mathrm{H} 1$ (data not shown). Mammalian PTPA increased twofold the activity of PPH21 toward both substrates in the $\operatorname{rrd} 1 \Delta / r r d 2 \Delta$ strain but decreased its activity in the wild-type strain, suggesting that mammalian PTPA can activate the PSer/P-Thr activity of the yeast catalytic subunit, but increased levels of RRD/PTPA affect catalytic activity negatively.

Despite the similar percentage of identical residues between mammalian PTPA and either one of the RRD proteins, deletion of $R R D 2$ caused a much larger decrease in the P-Ser/P-Thr activity of PP2A than did the deletion of the RRD1 gene (see Fig. 4B). We determined the individual activation potential of the RRD proteins on human or yeast $\mathrm{C}$ subunit by coexpressing HA-tagged human or yeast $\mathrm{C}$ subunit PPH21 with myc-tagged RRD1 or RRD2 in wild-type and $r r d 1 \Delta / r r d 2 \Delta$ strains. Immunoprecipitates of HA-tagged C subunits were then analyzed for catalytic activity toward phosphorylase a (Fig. 5B). Expression of RRD2 in the $r r d 1 \Delta / r r d 2 \Delta$ strain activated the yeast $C$ subunit almost up to wild-type levels and similarly activated mammalian $C$ subunit more than threefold. RRD1 expression was much less effective increasing the activity of either phosphatase catalytic subunit. Thus, the data suggest that RRD2 is significantly more active in vivo on both yeast and mammalian PP2A catalytic subunit.

Because PTPA and the catalytic subunit of PP2A interact weakly in mammalian cells (Fig. 1C), we evaluated the potential interaction between RRD2 and PPH21 in vivo. Immunoprecipitates of endogenous (untagged) PPH21 from lysates of wild-type and $r r d 1 \Delta / r r d 2 \Delta$ strains were probed for coprecipitated RRD proteins in immunoblot analysis. RRD2 interacted with the yeast PP2A C subunit, whereas RRD1 could not be detected in the PPH21 immunoprecipitate (Fig. 5C); furthermore, HAtagged PPH22 and HA-tagged human C coprecipitated RRD2 but not RRD1 in yeast (data not shown), indicating that the binding domains are conserved between yeast and man. The in vivo interaction between $C$ subunit and RRD2 and the ability of RRD2 to activate the 
A

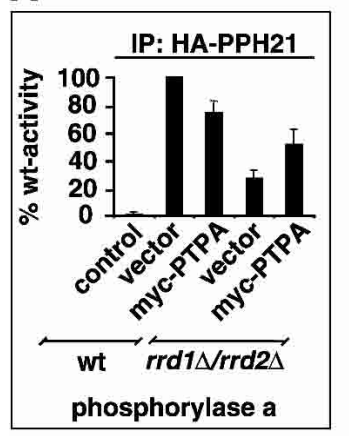

C

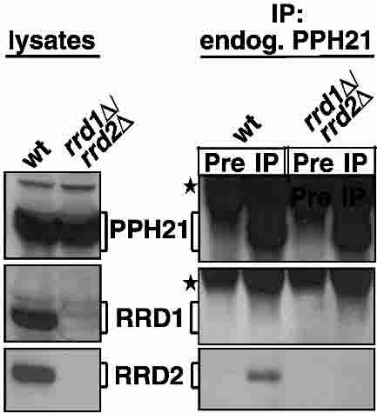

B

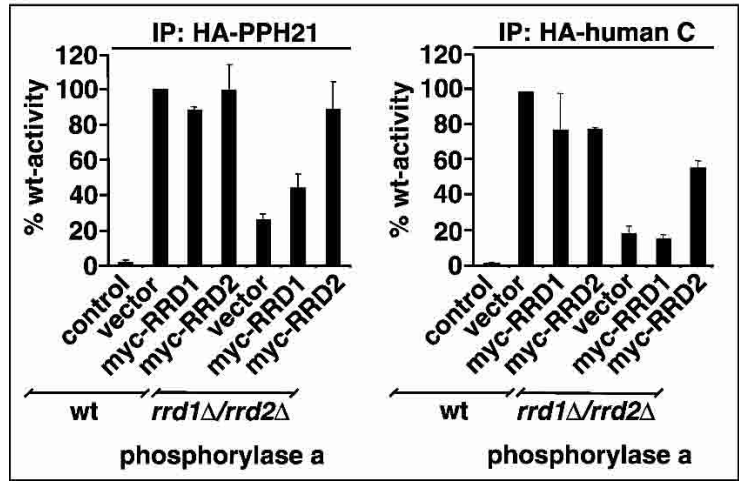

Figure 5. The functional interaction between RRD/PTPA and PP2A is evolutionarily conserved. (A) Anti-HA tag immunoprecipitates from lysates of wild-type and $r r d 1 \Delta / r r d 2 \Delta$ cells expressing HA-tagged PPH21 and myc-tagged mammalian PTPA (myc-PTPA) or containing empty vector (vector) were assayed for catalytic activity toward phosphorylase a. Control indicates an assay using anti-HA immunoprecipitates from lysates of wild-type cells expressing untagged PPH21 (see Materials and Methods for details). (B) Anti-HA tag immunoprecipitates from lysates of wild-type and $r r d 1 \Delta / r r d 2 \Delta$ cells expressing HA-tagged PPH21 (left graph) or HA-tagged human C subunit (right graph) together with myc-tagged RRD1 (myc-RRD1) or myc-tagged RRD2 (myc-RRD2) or containing empty vector (vector) were assayed for catalytic activity toward phosphorylase a. Control indicates an assay using anti-HA immunoprecipitates from lysates of wild-type cells containing only empty vectors (see Materials and Methods for details). (C) Endogenous PPH21 was immunoprecipitated from lysates of wild-type and $r r d 1 \Delta / r r d 2 \Delta$ cells with anti-PPH21 antibody serum (IP). Preimmune serum was used for control immunoprecipitations (Pre). Lysates (left) and immunoprecipitates (right) were separated on 10\% SDSPAGE and analyzed by immunoblotting with anti-PPH21, antiRRD1, and anti-RRD2 antibody. Asterisks indicate the heavy chain of anti-PPH21 antibody and other antibodies present in the rabbit serum.

P-Ser/P-Thr activity of PP2A indicate that PP2A C subunit is a direct target of the yeast PTPA homolog RRD2.

The requirement for $R R D / P T P A$ function is specific for the PP2A phosphatase family

Whereas RRD2 seems to target the PP2A C subunit physically as well as functionally, RRD1 is known to interact genetically and biochemically with SIT4, a PP2A-related phosphatase (Mitchell and Sprague 2001). To analyze whether RRD1 activates the catalytic activity of SIT4 in the same way that RRD2 activates the catalytic activity of the PP2A C subunit, we took advantage of the fact that SIT4 associates with several proteins, SAP155, SAP185, and SAP190, which are hyperphosphorylated in the absence of SIT4 (Luke et al. 1996), suggesting that they are SIT4 substrates. As an indirect measure of intracellular SIT4 activity, we expressed HA-tagged SAP155 or SAP185 in wild-type and RRD deletion strains and analyzed their migration pattern in SDS-PAGE and immunoblots. The electrophoretic mobility of HA-tagged SAP185 (Fig. 6A) and HA-tagged SAP155 (data not shown) decreased if RRD1 was lacking, suggesting that SIT4 activity was decreased in $\operatorname{rrd} 1 \Delta$ strains. The extent of the shift to slower migrating forms was less than in strains deleted for SIT4, indicating that SIT4 activity was not completely abolished in $\operatorname{rrd1\Delta }$ strains.

Next we asked whether the observed effect of RRD2 and RRD1 on catalytic activity is specific for PP2A and PP2A-like phosphatases such as SIT4, or whether the catalytic activity of distantly related serine/threonine protein phosphatases such as PP1 would also be decreased in the deletion strains. We expressed HA-tagged GLC7, the PP1 catalytic subunit, in the wild-type and mutant strains and tested the catalytic activity of immunoprecipitated GLC7 toward phosphorylase a (Fig. 6B). In contrast to the effect on PP2A/SIT4, the catalytic activity of GLC7 was increased in the single as well as the double $R R D$ deletion strains. Moreover, we were unable to detect an interaction between GLC7 and either one of
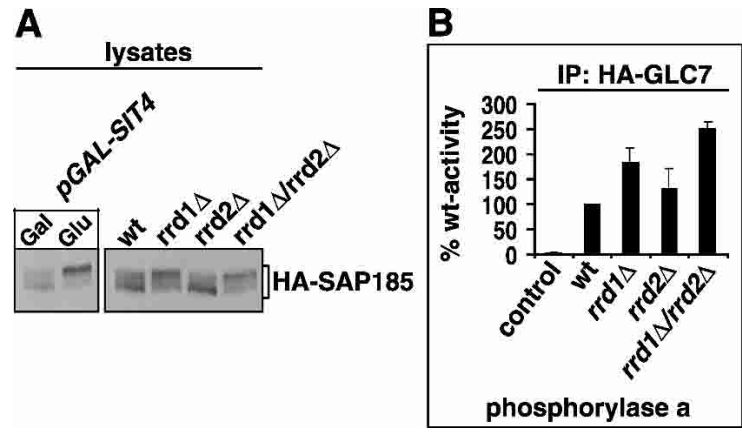

Figure 6. The requirement for RRD/PTPA function is specific for the PP2A phosphatase family. (A) pGAL-SIT4 cells in which the expression of SIT4 is under control of the GAL1 promoter were grown to log phase in CM dropout medium supplemented with glucose (Glu) or galactose (Gal). Lysates from these cultures as well as from logarithmic wild-type, $\operatorname{rrd1} \Delta, \operatorname{rrd} 2 \Delta$, and $r r d 1 \Delta / r r d 2 \Delta$ cells expressing HA-tagged SAP185 were prepared under denaturing conditions (trichloroacetic acid precipitation) and proteins were separated by $7.5 \%$ SDS-PAGE and analyzed by immunoblotting with anti-HA antibody. (B) Anti-HA tag immunoprecipitates from lysates of wild-type cells containing empty pYES2 vector (control) and of wild-type, $\operatorname{rrd1} 1 \Delta, \operatorname{rrd} d 2 \Delta$, and $r r d 1 \Delta / r r d 2 \Delta$ cells expressing HA-tagged GLC7 were assayed for phosphatase activity toward phosphorylase a (see Materials and Methods for details). 
the RRD proteins by probing the GLC7 immunoprecipitates from wild-type strains with specific antibodies (data not shown). Thus, the observed increase in GLC7 activity in the deletion strains might be a secondary effect of the low PP2A/SIT4 activity in these strains because it is known that there is regulatory cross-talk between these phosphatases.

Loss of RRD/PTPA function causes the synthesis of a PP2A C subunit with altered substrate specificity and metal ion dependence

PTPA was initially identified as an in vitro activity that stimulated in an $\mathrm{ATP} / \mathrm{Mg}^{2+}$-dependent manner the activity of purified PP2A on P-Tyr, using pNPP as a substrate (Cayla et al. 1990). To determine how the loss of PTPA function affects PP2A in vivo, we analyzed the catalytic activity of PPH21 complexes isolated from wild-type or $r r d 1 \Delta / r r d 2 \Delta$ strains toward pNPP. Surprisingly, loss of RRD function in vivo caused an eightfold increase of the pNPP activity of PPH21 (Fig. 7A), a phenotype opposite to that predicted from the in vitro results. Because pNPP is similar to P-Tyr and more bulky than P-Ser or P-Thr, its enhanced hydrolysis suggested that PPH21 synthesized in the absence of RRD proteins might be conformationally different from the wild-type protein. Misfolded proteins are recognized by the cellular quality control system, which tries to refold or targets the protein for degradation (Hohfeld et al. 2001). Interestingly, endogenous as well as HAtagged PPH21 and the human $\mathrm{C}$ subunit seemed to be less stable in an $r r d 1 \Delta / r r d 2 \Delta$ than in a wild-type strain, as shown by the appearance of degradation fragments in the immunoblot analysis of cell lysates from these strains (data not shown). Moreover, pulse-chase experiments revealed that the half-life of HA-tagged PPH21 decreased from $2.0 \mathrm{~h}$ in the wild type to $1.1 \mathrm{~h}$ in the $\operatorname{rrd} 1 \Delta / r r d 2 \Delta$ strain, supporting our assumption that the misfolded catalytic subunit is less stable in the deletion strain (Fig. 7B). In summary, loss of RRD functions resulted in the synthesis of a PP2A C subunit with reduced substrate specificity/activity and decreased stability.

PPH21 isolated from the $r r d 1 \Delta / r r d 2 \Delta$ strain displayed reduced substrate specificity very similar to bacterially expressed PP1. The reduced substrate specificity of recombinant PP1 has been suggested to be due to a conformationally altered active site allowing accommodation of a bulky P-Tyr substrate (Egloff et al. 1995; MacKintosh et al. 1996). The more flexible active site seems to be also responsible for the easy loss of metal ions from the active site, as indicated by the metal dependent activation of the recombinant enzyme. To determine whether the P-Ser/P-Thr activity of PPH21 isolated from the $r r d 1 \Delta / r r d 2 \Delta$ strain can be reactivated by metal ions in vitro, we incubated immunoprecipitates of HA-tagged $\mathrm{PPH} 21$ from lysates of the wild-type and the $r r d 1 \Delta / r r d 2 \Delta$ strain with different bivalent metal ions for $30 \mathrm{~min}$ and subsequently measured their catalytic activity toward
A
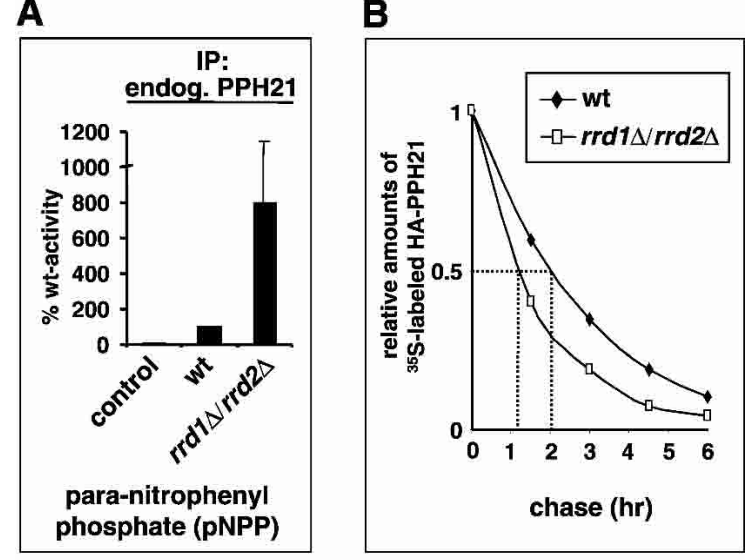

C

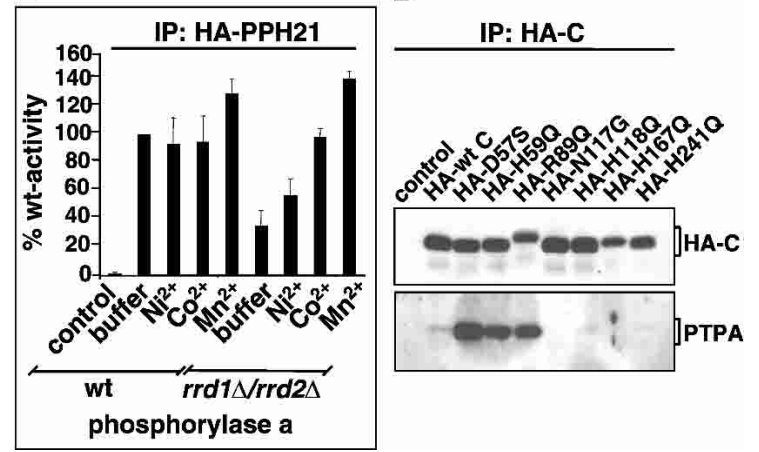

Figure 7. Loss of RRD/PTPA function causes the synthesis of a PP2A C subunit with altered substrate specificity and metal ion dependence. $(A)$ Endogenous $C$ subunit was immunoprecipitated from lysates of wild-type and $r r d 1 \Delta / r r d 2 \Delta$ cells with monoclonal C subunit antibody 7A6. Immunopurified PP2A complexes were assayed for catalytic activity toward para-nitrophenyl phosphate (pNPP; see Materials and Methods for details). (B) Pulse-chase analysis of HA-PPH21. Wild-type and $r r d 1 \Delta / r r d 2 \Delta$ cells expressing HA-tagged $\mathrm{PPH} 21$ from the vector pYX142 were subjected to pulse-chase experiments as described in Materials and Methods. Immunoprecipitation was carried out with anti-HA antibody. The amount of ${ }^{35}$ S-labeled HAPPH21 was normalized to the amount of total immunoprecipitated protein. $(C)$ Anti-HA-PPH21 immunoprecipitates from lysates of wild-type and $r r d 1 \Delta / r r d 2 \Delta$ cells were split into equal parts and incubated with phosphatase assay buffer (buffer) or buffer containing $1 \mathrm{mM}$ of the indicated metal ions for $30 \mathrm{~min}$ at $30^{\circ} \mathrm{C}$. Phosphatase activity was assayed toward phosphorylase $\mathrm{a}$ in the presence of metal ions (see Materials and Methods for details). (D) Anti-HA immunoprecipitates from lysates of NIH3T3 cells containing empty pBabe-puro vector (control) or stably expressing HA-tagged human wild-type or mutant C subunits were analyzed by $10 \%$ SDS-PAGE and immunoblotting with anti-HA and anti-PTPA antibody.

serine-/threonine-phosphorylated phosphorylase a (Fig. 7C) or cdc2-phosphorylated histone H1 (data not shown). Incubation with $\mathrm{Co}^{2+}$ or $\mathrm{Mn}^{2+}$ stimulated the P-Ser/PThr activity of PPH 21 from the deletion strain up to or above the levels of the wild-type strain enzyme, whereas $\mathrm{Ni}^{2+}$ incubation was less effective in this regard. The catalytic activity of the wild-type strain enzyme was 
slightly activated by $\mathrm{Mn}^{2+}$ and inhibited a little by $\mathrm{Ni}^{2+}$ and $\mathrm{Co}^{2+}$. The in vitro reactivation of the catalytically impaired $r r d 1 \Delta / r r d 2 \Delta$ enzyme by certain metal ions suggested that metal ions were not tightly bound to the active site in the absence of RRD/PTPA function. Along with the other properties described so far for the $r r d 1 \Delta$ / rrd2s enzyme, the metal dependency suggests that the catalytic core was not correctly folded in an $r r d 1 \Delta / r r d 2 \Delta$ strain.

\section{Mutants of metal-binding residues show increased association with mammalian PTPA}

Mutation of the predicted metal-binding residue H59 in the PP2A C subunit stabilizes the interaction with several cellular proteins, one of which we identified as PTPA (Fig. 1). To determine whether mutation of other predicted metal coordinating residues also enhanced the interaction with PTPA, we tested the ability of additional mutants for complex formation with endogenous PTPA. We generated NIH3T3 fibroblasts stably expressing the individual $\mathrm{C}$ subunit mutant and analyzed immunoprecipitates of HA-tagged mutants for coprecipitated endogenous PTPA (Fig. 7D). The active site mutants, D57S, H59Q, and R89Q, bound increased amounts of PTPA, indicating that the ability for increased binding of PTPA was not unique to the mutant H59S. On the other hand, stable association with PTPA was not due solely to the loss of catalytic activity because substitution mutations of amino acids N117, H118, H167, or $\mathrm{H} 241$, which all result in the loss of catalytic activity, did not increase the interaction between the mutant proteins and PTPA. Based on the PP1 crystal structure, the PP2A residues D57 and $\mathrm{H} 59$ are predicted to bind metal ion 1 (M1); the residues N117, H167, and H241 to bind metal ion 2 (M2); R89 to coordinate the substrate-phosphate; and $\mathrm{H} 118$ to protonize the leaving group oxygen (schematic illustrated in Fig. 8; Barford 1996). Interestingly, only mutation of M1-coordinating residues D57 and $\mathrm{H} 59$ or of R89, a residue in close proximity to M1, led to the stable interaction with PTPA. Together with the data on the reactivation of $r r d 1 \Delta / r r d 2 \Delta$ made PP2A by bivalent metal ions, these findings indicate a potential role of RRD/PTPA in the generation of a PP2A cata- lytic core with a high affinity conformation for metal ions.

\section{Discussion}

Here we present evidence suggesting the molecular mechanism of action of RRD/PTPA in vivo. For over a decade, the only function assigned to PTPA was its ability to activate transiently and in vitro the phosphotyrosyl phosphatase activity of the mono- and dimeric forms of PP2A (Goris et al. 1988; Cayla et al. 1990). The role of PTPA in the activation process remained mysterious because the essential cofactors of the reaction, ATP and $\mathrm{Mg}^{2+}$, are able on their own to activate the phosphotyrosyl phosphatase activity of PP2A. The physiological significance of the transient phosphotyrosyl activity of PP2A, a bona fide PSTP, was unclear, but the high degree of sequence conservation of PTPA hinted at a very important and conserved function for PTPA. We show that the RRD proteins are required for generation of an active and P-Ser/P-Thr-specific catalytic subunit of PP2A as well as for generation of active SIT4, a PP2A-related phosphatase, in vivo. Our data provide a potential explanation for the pleiotropy and severity of the phenotypes of the $R R D$ deletion strains. The importance of RRD/ PTPA function is further underlined by our finding that PTPA is essential for survival of mammalian cells.

Could the in vitro function of PTPA as a phosphotyrosyl activator account for the observed phenotypes of the $R R D$ deletion strains? Deletion of $R R D 1$ or $R R D 2$ is associated with hypersensitivity to spindle depolymerizing drugs or resistance to the immunosuppressant rapamycin, phenotypes that are also observed in strains deleted in the regulatory, substrate-targeting B-type subunits of PP2A, therefore lacking B subunit dependent dephosphorylation (Minshull et al. 1996). The in vitro activity of PTPA as a phosphotyrosyl phosphatase activator affects only the mono- and dimeric forms of PP2A but not heterotrimeric complexes and thus cannot provide an explanation for the defective spindle assembly checkpoint of the $R R D$ deletion strains. Our data, however, show that the loss of RRD/PTPA caused a drop in the P-Ser/P-Thr-specific catalytic activity of PP2A, including trimeric complexes with $\mathrm{B}$ subunit. Thus, we
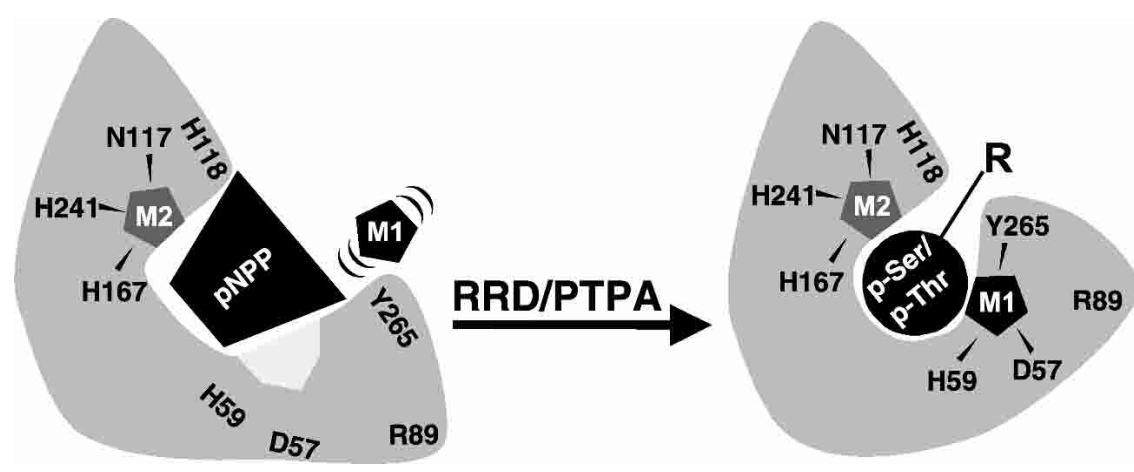

Figure 8. Model of RRD/PTPA function. Para-nitrophenyl phosphate (pNPP) represents a bulky P-Tyr-like substrate. (P-SerR) phospho-serine substrate; (P-Thr-R) phospho-threonine substrate. M1 and M2 indicate metal ion 1 and metal ion 2, respectively, located in the catalytic core of the catalytic subunit of PP2A. The indicated amino acid residues represent amino acids of the PP2A catalytic subunit that are required for metal ion coordination and catalysis. (D) Asp; (H) His; (N) Asn; (R) $\operatorname{Arg}_{;}(\mathrm{Y})$ Tyr. See Results and Discussion sections for details. 
propose that, in vivo, RRD/PTPA does not solely function as an intracellular phosphotyrosyl activator of PP2A.

Loss of the RRD proteins resulted in the generation of a PP2A catalytic subunit with altered biochemical properties, which indicated that the conformation of the enzyme might be different from a wild-type enzyme. According to our model (Fig. 8), the conformationally relaxed active site permits entry of the bulky P-Tyr-like substrate pNPP and binds metal ions less tightly, as shown by the metal-dependent reactivation of the enzyme. Our findings are reminiscent of the data on the conformational differences between bacterially expressed and native PP1 (MacKintosh et al. 1996). The crystal structure of bacterially expressed PP1 indicated that the $\beta 12-\beta 13$ loop containing the absolutely conserved residue Y272 (Y265 in PP2A) might play an important role in the switch from the metal-dependent to the native forms (Egloff et al. 1995; Connor et al. 1999). It has been suggested that motion of the loop toward the active site compresses the active site channel, excluding the bulky P-Tyr side chain from the active site, and increases the affinity for $\mathrm{M} 1$ by coordinating $\mathrm{M} 1$ to the shifted side chain of Y272 (Y265). The switch of PP1 into its native conformation is carried out by inhibitor-2 (I-2) in a chaperone-like process (Alessi et al. 1993; MacKintosh et al. 1996). Our data suggest that RRD/PTPA might play a similar role for generation of the native conformation of the PP2A and also potentially the SIT4 active site.

Consistent with a role of RRD/PTPA at the active site of PP2A, we found that mutation of the M1 coordinating residues D57 and $\mathrm{H} 59$ as well as of R89 stabilized the interaction between mammalian PTPA and the C subunit mutants, whereas only a weak and transient interaction could be detected between mammalian wild-type C subunit and endogenous PTPA. Stabilization of the interaction was specifically associated with mutation of these residues but not of the predicted ligands of the second metal ion. The mutants D57N and H59Q are known to be catalytically inactive because the metal ion M1, which is essential for catalysis, is most likely lost from the active site (Ogris et al. 1999b). Thus, it is tempting to speculate that the interaction with PTPA is stabilized because the loss of metal ion M1 might not locate the $\beta 12-\beta 13$ loop in its final position at the active site and thus the conformational switch cannot be completed.

The P-Ser/P-Thr-specific activity of PP2A in an $r r d 1 \Delta /$ rrd2s strain was not abolished but dropped on average down to $25 \%$ of the wild-type strain activity. The phenotype of the $r r d 1 \Delta / r r d 2 \Delta$ strain, however, is at least as severe as the one of a strain entirely lacking PP2A activity (Sneddon et al. 1990; Ronne et al. 1991). Thus, the decrease of PP2A activity in an $r r d 1 \Delta / r r d 2 \Delta$ strain cannot alone account for the observed phenotype. An additional reason could be that the altered/broadened substrate specificity of PP2A caused the intracellular dephosphorylation of tyrosine phosphorylated substrates that are usually not substrates of PP2A. However, although the $r r d 1 \Delta / r r d 2 \Delta$ strain PP2A was hyperactive to- ward pNPP, it did not show an increased activity toward a phosphotyrosyl-peptide substrate TSTEPQpYQPGENL (data not shown), making this possibility less likely. In addition, overexpression of PPH22 partially suppresses the $r r d 1 \Delta / r r d 2 \Delta$ phenotype (Rempola et al. 2000); if altered substrate specificity is one of the causes for the phenotype, then a surplus of PP2A should, if anything, worsen rather than suppress the phenotype.

A more likely possibility is that loss of the RRD proteins affects other proteins in addition to $\mathrm{PPH} 21$ and PPH22. A recent study implicated SIT4, a PP2A-related phosphatase, as a potential target of RRD1 and maybe also RRD2 (Mitchell and Sprague 2001). Our data provide first experimental proof for the intracellular role of RRD1 as an activator/regulator of SIT4 catalytic activity, providing a potential explanation for the genetic interaction between SIT4 and RRD1 on a molecular level. It is less clear, however, whether RRD2 has a role in regulating SIT4. The interaction between SIT4 and RRD2 was only shown by an in vitro binding assay using unphysiological amounts of proteins (Mitchell and Sprague 2001). Our data suggest that PPH21 and PPH22 might be the targets of RRD2. RRD2 but not RRD1 physically interacted in vivo with PPH21, PPH22, and human PP2A C subunit expressed in yeast, indicating the evolutionary conservation of the interaction. Loss of RRD2 function caused a larger decrease of PP2A catalytic activity than the loss of RRD1, and, reversibly, recombinant expression of RRD2 in an $r r d 1 \Delta / r r d 2 \Delta$ strain regenerated PP2A activity more efficiently than RRD1. We obtained similar results for the human or the yeast $\mathrm{C}$ subunit pointing to a conserved regulatory role of RRD/PTPA. The observed partial redundancy in the activation of PP2A by RRD1 or RRD2 might be due to the high degree of conservation of the phosphatases and the RRD/PTPAs. However, our data do not exclude the possibility that, apart from RRD2, RRD1 plays a role on its own in the biogenesis of active and specific PP2A.

Is RRD/PTPA required for the controlled generation of PP2A catalytic activity in vivo? Substrate specificity and targeting of PP2A is achieved through the interaction of $\mathrm{C}$ subunit with different regulatory subunits. Binding of the B-type subunits as well as stable association with the A subunit seems to require methylation of the C-terminal leucine of the C subunit (Mumby 2001). Until methylation occurs and complexes assemble, newly synthesized $\mathrm{C}$ subunit would be a highly active but unspecific phosphatase. Thus, we expected that, in a strain with a deletion of the methyltransferase gene PPM1, the free C subunit would possess a several-fold higher activity toward phosphorylase a than PP2A from a wild-type strain. However, conversely to the prediction, the catalytic activity was reduced to $40 \%$ of the wild-type levels, suggesting that methylation is required for efficient activation of PP2A. Interestingly, RRD2 was still able to interact with $\mathrm{PPH} 21$ in the ppm1s strain, indicating that, in contrast to the $\mathrm{A}$ and $\mathrm{B}$ regulatory subunits, methylation does not seem to be a prerequisite for the interaction with RRD2 (data not shown). In addition, low-level overexpression of RRD/PTPA in a wild-type strain inhibited 
catalytic activity of PPH21, indicating a potential dual function of RRD/PTPA as an activator and inhibitor of PP2A in vivo. It is tempting to speculate that RRD/ PTPA proteins, with their activating/inhibitory functions, might be part of a cascade regulating timed activation of PP2A catalytic activity in vivo.

\section{Materials and methods}

\section{Plasmids and mutagenesis}

Plasmids used in this study are listed in Table 1. The coding region of mouse PTPA was amplified by reverse transcriptase PCR (RT-PCR) using RNA of NIH3T3 cells as template. The $R R D 1, R R D 2, G L C 7$, and SIT4 coding region was amplified by PCR using yeast genomic DNA as template. Detailed cloning strategies of the individual constructs can be obtained on request.

Mammalian tissue culture, gene transfer, and metabolic labeling

NIH3T3 and HeLa cells were grown in Dulbecco's Modified Eagle's Medium (DMEM, Sigma) supplemented with 10\% (v/v) calf serum (CS, Life Technologies, Inc.) and Penicillin-Streptomycin Solution (Sigma). The murine viral-mediated transfer system described by Pear et al. (1993) was used to transfer and express exogenous genes in NIH3T3 cells. HeLa cells were transfected using Effectene Transfection Reagent (Qiagen). Puromycin (Sigma) was used at a concentration of $5 \mu \mathrm{g} / \mathrm{mL}$ for NIH3T3 cells and $0.8 \mu \mathrm{g} / \mathrm{mL}$ for HeLa cells. G418-sulphate (Life Technologies, Inc.) was added to NIH3T3 cells at a concentration of $400 \mu \mathrm{g} / \mathrm{mL}$. Metabolic labeling of NIH3T3 cells with ${ }^{35} \mathrm{~S}$-methionine (NEN) was performed as described (Ogris et al. 1999a).

\section{Yeast strains, growth media, and gene disruption}

S. cerevisiae strains used in this study are listed in Table 2. All strains are descendants of S288C, which has been shown to contain the SSD1-V allele (Sutton et al. 1991). Yeast cells were grown in rich medium (YPD) or in complete minimal (CM) dropout medium supplemented with either $2 \%$ glucose or $2 \%$ galactose and $0.1 \%$ raffinose and prepared according to standard recipes. PCR-based gene targeting was carried out as described using the modules HIS3MX6 (Wach et al. 1997) and HIS3MX6PGAL1-3HA (Longtine et al. 1998), respectively. The strain $p G A L-R R D 1 /$ rrd2 $\Delta$ expresses $3 \times \mathrm{HA}$-tagged RRD1 under the

Table 1. Plasmids

\begin{tabular}{|c|c|c|}
\hline Plasmid & Characteristics/expression product & Source \\
\hline \multicolumn{3}{|c|}{ Mammalian expression plasmid } \\
\hline pcDNA I Amp[HA-wt C] & HA-tagged human wt $\mathrm{C}$ subunit & (Ogris et al. 1999a) \\
\hline pBabepuro & & (Morgenstern and Land 1990 \\
\hline pBabepuro[HA-wt C] & HA-tagged human wt $\mathrm{C}$ subunit & This study \\
\hline pBabepuro[HA-D57S] & HA-tagged human $\mathrm{C}$ subunit mutant D57S & This study \\
\hline pBabepuro[HA-H59Q] & HA-tagged human $\mathrm{C}$ subunit mutant $\mathrm{H} 59 \mathrm{Q}$ & This study \\
\hline pBabepuro[HA-H59S] & HA-tagged human C subunit mutant H59S & This study \\
\hline pBabepuro[HA-R89Q] & HA-tagged human C subunit mutant R89Q & This study \\
\hline pBabepuro[HA-N117G] & HA-tagged human C subunit mutant N117G & This study \\
\hline pBabepuro[HA-H118Q] & HA-tagged human C subunit mutant $\mathrm{H} 118 \mathrm{Q}$ & This study \\
\hline pBabepuro[HA-H167Q] & HA-tagged human $\mathrm{C}$ subunit mutant $\mathrm{H} 167 \mathrm{Q}$ & This study \\
\hline pBabepuro[HA-H241Q] & HA-tagged human C subunit mutant $\mathrm{H} 241 \mathrm{Q}$ & This study \\
\hline pBabeneo & & (Morgenstern and Land 1990 \\
\hline pBabeneo[HA-wt C] & HA-tagged human wt $\mathrm{C}$ subunit & This study \\
\hline pBabeneo[HA-H59S] & HA-tagged human C subunit mutant H59S & This study \\
\hline pBabe-puro[myc-PTPA] & Myc-tagged mouse PTPA & This study \\
\hline pBabe-Retro ${ }_{\text {Katze }}$ & Puromycin resistance marker, green fluorescence protein (GFP) & This study \\
\hline \multicolumn{3}{|l|}{ RNA interference plasmid } \\
\hline pSUPER & & Oligoengine \\
\hline pSUPER-NS-PTPA & NONSENSE-sequence 5'-ATACCGCTCAGTAGCGACA-3' & This study \\
\hline pSUPER-PTPA & PTPA-targeting sequence 5'-GTTCCCTGTGATCCAGCAC-3' & This study \\
\hline \multicolumn{3}{|l|}{ Yeast expression plasmid } \\
\hline pyx 142 & CEN LEU2 TPI promoter & R\&D Systems \\
\hline pyx $142[$ myc-PTPA] & Myc-tagged mouse PTPA & This study \\
\hline pyx142[HA-human C] & HA-tagged human wt $\mathrm{C}$ subunit & This study \\
\hline pyx 142[HA-PPH21] & HA-tagged PPH21 & This study \\
\hline pyx 112 & CEN URA3 TPI promoter & R\&D Systems \\
\hline pyx112[myc-RRD1] & Myc-tagged RRD1 & This study \\
\hline pyx112[myc-RRD2] & Myc-tagged RRD2 & This study \\
\hline pRS316[PPH21] & PPH21 & (Wei et al. 2001) \\
\hline pRS316[HA-PPH21] & HA-tagged $\mathrm{PPH} 21$ & (Wei et al. 2001) \\
\hline pYES2 & $U R A 32 \mu$ ori $G A L 1$ promoter & Invitrogen \\
\hline pYES2[HA-SIT4] & HA-tagged SIT4 & This study \\
\hline pYES2[HA-GLC7] & HA-tagged GLC7 & This study \\
\hline YCp50[HA-SAP185] & HA-tagged SAP185 & (Luke et al. 1996) \\
\hline YCp50[HA-SAP155] & HA-tagged SAP155 & (Luke et al. 1996) \\
\hline
\end{tabular}

Amino acid residues (D) Asp; (G) Gly; (H) His; (N) Asn; (Q) Gln; (R) Arg; (S) Ser. 
Fellner et al.

Table 2. Yeast strains

\begin{tabular}{|c|c|c|}
\hline Strain & Genotype & Source \\
\hline BY4741 & 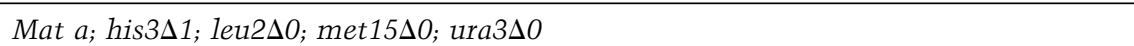 & $\overline{\text { Euroscarf }}$ \\
\hline $\operatorname{rrd1} \Delta$ & 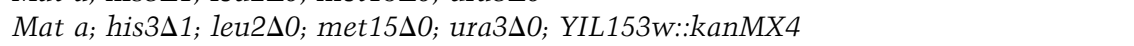 & Euroscarf \\
\hline $\operatorname{rrd2\Delta }$ & 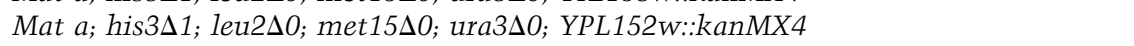 & Euroscarf \\
\hline ppm1s & 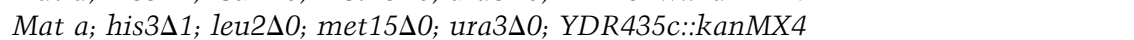 & Euroscarf \\
\hline$r r d 1 \Delta / r r d 2 \Delta$ & 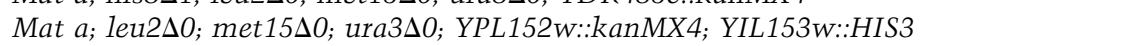 & This study \\
\hline$p G A L-R R D 1 / r r d 2 \Delta$ & 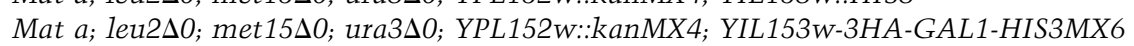 & This study \\
\hline pGAL-SIT4 & 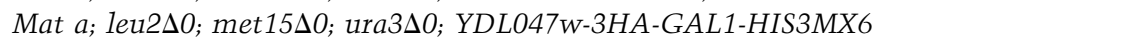 & This study \\
\hline
\end{tabular}

control of the glucose-repressible and galactose-inducible GAL1 promotor. For all experiments shown in this study, $p G A L$ $R R D 1 / r r d 2 \Delta$ cells were grown on glucose-containing plates or glucose-containing liquid medium to ensure constitutive repression of RRD1 expression.

Preparation of cell lysates, immunoprecipitation, and immunoblotting

Preparation of whole-cell lysates of mammalian cells was carried out as described (Ogris et al. 1999a) except that the NP40 lysis buffer was supplemented with Complete Protease Inhibitor Cocktail (Roche). To generate whole-cell lysates of yeast cells, $50 \mathrm{OD}_{600}$ equivalents of logarithmically growing cells were washed once in cold $\mathrm{ddH}_{2} \mathrm{O}$ and then resuspended in 600 $\mu \mathrm{L}$ cold yeast lysis buffer [50 mM Tris- $\mathrm{Cl}$ at $\mathrm{pH} 7.6,150 \mathrm{mM}$ $\mathrm{NaCl}, 1 \%(\mathrm{v} / \mathrm{v})$ Triton X-100, and $1 \mathrm{mM}$ EDTA] supplemented with $1 \mathrm{mM}$ phenylmethylsulfonyl fluoride (PMSF), 0.03 units/ $\mathrm{mL}$ aprotinin (Sigma), and Complete. For the experiment shown in Figure 4A, the yeast lysis buffer was supplemented with 50 $\mathrm{mM}$ sodium fluoride, $20 \mathrm{mM}$ sodium pyrophosphate, $1 \mathrm{mM}$ sodium ortho-vanadate, $100 \mathrm{mM} \mathrm{B-glycerophosphate,} \mathrm{and} 4$ mM EDTA. Cells were lysed in the presence of $400 \mu \mathrm{L}$ of ice cold glass beads (425-600 $\mu \mathrm{m}$, nitric acid washed, Sigma) by mechanical disruption at $1.400 \mathrm{rpm}$ for $1 \mathrm{~h}$ at $4^{\circ} \mathrm{C}$.

Myc- or HA-tagged proteins were immunoprecipitated with anti-HA (12CA5) or anti-myc (9E10) antibody cross-linked to BSA-coated protein-A-sepharose (Pharmacia Biotech). Endogenous PPH21 was immunopurified with polyclonal anti-PPH2 1 antibody (raised against residues 1-186 of PPH21) plus proteinA-sepharose or with mouse monoclonal anti-C subunit antibody (clone 7A6, Upstate Biotechnology) plus a mixture of protein-A/-G-sepharose (Pharmacia Biotech). Following incubation for $1 \mathrm{~h}$ at $4^{\circ} \mathrm{C}$ under gentle agitation, coimmunoprecipitates were washed once with $1 \mathrm{~mL}$ ice cold lysis buffer and three times with $1 \mathrm{~mL}$ Tris-buffered saline (TBS; $25 \mathrm{mM}$ Tris- $\mathrm{HCl}$ at $\mathrm{pH} 7.4,137 \mathrm{mM} \mathrm{NaCl}, 2.7 \mathrm{mM} \mathrm{KCl}$ ). Immunoprecipitates were analyzed by $7.5 \%$ or $10 \%$ SDS-PAGE or two-dimensional gel electrophoresis (Ogris et al. 1999a). Immunodetection was performed with mouse monoclonal anti-HA (clone 16B12, 1:5.000, BAbCO), anti-myc [clone 9E10, 1:1000 (Evan et al. 1985)], rabbit polyclonal anti-PPH21 (1:10.000), rabbit polyclonal anti-PTPA (raised against full length mouse PTPA, 1:10.000), rabbit polyclonal anti-C subunit (raised against residues 288-303 of the human PP2A C subunit, 1:10.000), rabbit polyclonal anti-TPD3 (Wei et al. 2001), rabbit polyclonal anti-CDC55 (Wei et al. 2001), rabbit polyclonal anti-RRD1 (raised against residues 1-140 of RRD1, 1:10.000), mouse monoclonal anti-RRD2 (clone 4G9, 1: 200), and rabbit polyclonal Anti-HSP70 (Upstate Biotechnology) antibodies.

For pulse-chase experiments, $75 \mathrm{OD}_{600}$ equivalents of logarithmically growing yeast cells were harvested and washed two times in pulse medium (CM medium without methionine).
Cells were resuspended in $10 \mathrm{~mL}$ prewarmed pulse medium, grown for $15 \mathrm{~min}$ at $30^{\circ} \mathrm{C}$, and then labeled for $10 \mathrm{~min}$ by adding $8 \mu \mathrm{Ci} / \mathrm{OD}_{600}$ of ${ }^{35} \mathrm{~S}$-methionine (ICN). Chase period was performed in $150 \mathrm{~mL}$ fresh, prewarmed chase medium (CM medium $+1 \mathrm{mg} / \mathrm{mL}$ methionine). $10 \mathrm{OD}_{600}$ equivalents of yeast cells were removed at time points $0,1.5,3,4.5$, and $6 \mathrm{~h}$ and washed with ice-cold $10 \mathrm{mM} \mathrm{NaN}_{3}$. Cell lysis and immunoprecipitations were performed as described earlier. The amount of labeled protein was determined using a PhosphorImager and normalized to the amount of total immunoprecipitated protein, as determined by immunoblotting. The indicated half-life of HA-tagged PPH21 represents the average value of three independent experiments.

\section{Protein phosphatase assays}

Phosphatase activity of PP2A immunoprecipitates was assayed toward ${ }^{32} \mathrm{P}$-labeled phosphorylase $\mathrm{a},{ }^{32} \mathrm{P}$-labeled histone $\mathrm{H} 1,{ }^{32} \mathrm{P}$ labeled RCM-lysozyme, and para-nitrophenyl phosphate (pNPP, Sigma). Histone HI was phosphorylated by cdc2 protein kinase (New England Biolabs, Inc.) and RCM-lysozyme by cAMP-dependent protein kinase (Promega). Catalytic activity of PP2A was also measured using the Ser/Thr phosphatase assay kit 1 and the protein tyrosine phosphatase assay kit 2 (Upstate Biotechnology). The assay values were normalized to the amount of the respective immunoprecipitated protein as determined by immunoblot analysis and are presented as a percentage of the activity for PP2A complexes (or GLC7 in Fig. 6B) purified from the wild-type strain, which was set $100 \%$.

\section{Immunofluorescence staining}

Immunofluorescence staining was carried out as described (Moreno et al. 2001) except that blocking was done for $30 \mathrm{~min}$ in $1 \%(w / v)$ BSA/PBS. Anti-ACTIVE Caspase-3 pAb (Promega) and anti-rabbit IgG Alexa 594 Dye (Molecular Probes) were diluted $1: 1.000,1: 5.000$, respectively, in $0.1 \%(\mathrm{w} / \mathrm{v}) \mathrm{BSA} / \mathrm{PBS}$. DNA staining was performed with DAPI and samples were mounted using DAKO Fluorescent Mounting Medium (DAKO Corporation). Samples were analyzed by confocal and two photon microscopy using the Leica TCS System (Leica Microsystems).

\section{Acknowledgments}

We thank Kim Arndt, David Pallas, and Shirish Shenolikar for expression plasmids; Christoph Schüller, Yasmine Mamnun, Hubert Wolfger, Bettina Bauer, Karl Kuchler, and Manfred Koranda for help and advice on yeast methods; and Katharina Maderböck for generation of pBabe-Retro Katze . We are grateful to Patrick Hogan, Anjana Rao, and David Virshup for critical reading of the manuscript. This work was supported by grants from 
the Austrian Science Foundation (FWF MOB-P13707 and FWF P15685) and from the Herzfelder Familienstiftung.

The publication costs of this article were defrayed in part by payment of page charges. This article must therefore be hereby marked "advertisement" in accordance with 18 USC section 1734 solely to indicate this fact.

\section{References}

Alessi, D.R., Street, A.J., Cohen, P., and Cohen, P.T. 1993. Inhibitor-2 functions like a chaperone to fold three expressed isoforms of mammalian protein phosphatase-1 into a conformation with the specificity and regulatory properties of the native enzyme. Eur. J. Biochem. 213: 1055-1066.

Barford, D. 1996. Molecular mechanisms of the protein serine/ threonine phosphatases. Trends Biochem. Sci. 21:407412.

Beck, T. and Hall, M.N. 1999. The TOR signalling pathway controls nuclear localization of nutrient-regulated transcription factors. Nature 402: 689-692.

Brummelkamp, T.R., Bernards, R., and Agami, R. 2002. A system for stable expression of short interfering RNAs in mammalian cells. Science 296: $550-553$.

Burchell, A. and Cohen, P. 1978. Is phosphorylase phosphatase a manganese metalloenzyme? Biochem. Soc. Trans. 6: 220222.

Cai, L., Chu, Y., Wilson, S.E., and Schlender, K.K. 1995. A metal-dependent form of protein phosphatase 2A. Biochem. Biophys. Res. Commun. 208: 274-279.

Cayla, X., Goris, J., Hermann, J., Hendrix, P., Ozon, R., and Merlevede, W. 1990. Isolation and characterization of a tyrosyl phosphatase activator from rabbit skeletal muscle and Xenopus laevis oocytes. Biochemistry 29: 658-667.

Cohen, P. 1989. The structure and regulation of protein phosphatases. Annu. Rev. Biochem. 58: 453-508.

Connor, J.H., Kleeman, T., Barik, S., Honkanen, R.E., and Shenolikar, S. 1999. Importance of the $\beta 12-\beta 13$ loop in protein phosphatase- 1 catalytic subunit for inhibition by toxins and mammalian protein inhibitors. J. Biol. Chem. 274: 2236622372.

Di Como, C.J. and Arndt, K.T. 1996. Nutrients, via the Tor proteins, stimulate the association of Tap42 with type 2A phosphatases. Genes \& Dev. 10: 1904-1916.

Egloff, M.P., Cohen, P.T., Reinemer, P., and Barford, D. 1995. Crystal structure of the catalytic subunit of human protein phosphatase 1 and its complex with tungstate. J. Mol. Biol. 254: 942-959.

Evan, G.I., Lewis, G.K., Ramsay, G., and Bishop, J.M. 1985. Isolation of monoclonal antibodies specific for human c-myc proto- oncogene product. Mol. Cell. Biol. 5: 3610-3616.

Goris, J., Pallen, C.J., Parker, P.J., Hermann, J., Waterfield, M.D., and Merlevede, W. 1988. Conversion of a phosphoseryl/ threonyl phosphatase into a phosphotyrosyl phosphatase. Biochem. J. 256: 1029-1034.

Hohfeld, J., Cyr, D.M., and Patterson, C. 2001. From the cradle to the grave: Molecular chaperones that may choose between folding and degradation. EMBO Rep. 2: 885-890.

Hsiao, K.J., Sandberg, A.R., and Li, H.C. 1978. The role of ATP and divalent cations in the regulation of a cardiac phosphorylase phosphatase (phosphoprotein phosphatase) of $\mathrm{Mr}=35,000$. J. Biol. Chem. 253: 6901-6907.

Jiang, Y. and Broach, J.R. 1999. Tor proteins and protein phosphatase $2 \mathrm{~A}$ reciprocally regulate Tap42 in controlling cell growth in yeast. $E M B O J$. 18: 2782-2792.

Longtine, M.S., McKenzie III, A., Demarini, D.J., Shah, N.G.,
Wach, A., Brachat, A., Philippsen, P., and Pringle, J.R. 1998. Additional modules for versatile and economical PCR-based gene deletion and modification in Saccharomyces cerevisiae. Yeast 14: 953-961.

Luke, M.M., Della Seta, F., Di Como, C.J., Sugimoto, H., Kobayashi, R., and Arndt, K.T. 1996. The SAP, a new family of proteins, associate and function positively with the SIT4 phosphatase. Mol. Cell. Biol. 16: 2744-2755.

MacKintosh, C., Garton, A.J., McDonnell, A., Barford, D., Cohen, P.T., Tonks, N.K., and Cohen, P. 1996. Further evidence that inhibitor- 2 acts like a chaperone to fold PP1 into its native conformation. FEBS Lett. 397: 235-238.

Minshull, J., Straight, A., Rudner, A.D., Dernburg, A.F., Belmont, A., and Murray, A.W. 1996. Protein phosphatase 2a regulates MPF activity and sister chromatid cohesion in budding yeast. Curr. Biol. 6: 1609-1620.

Mitchell, D.A. and Sprague, G.F. 2001. The phosphotyrosyl phosphatase activator, Ncslp (Rrd1p), functions with Cla4p to regulate the G(2)/M transition in Saccharomyces cerevisiae. Mol. Cell. Biol. 21: 488-500.

Moreno, C.S., Lane, W.S., and Pallas, D.C. 2001. A mammalian homolog of yeast mobl is both a member and a putative substrate of striatin family-protein phosphatase $2 \mathrm{a}$ complexes. J. Biol. Chem. 276: 24253-24260.

Morgenstern, J.P. and Land, H. 1990. Advanced mammalian gene transfer: High titre retroviral vectors with multiple drug selection markers and a complementary helper-free packaging cell line. Nucleic Acids Res. 18: 3587-3596.

Mumby, M. 2001. A new role for protein methylation: Switching partners at the phosphatase ball. SCi. STKE 79: PE1.

Ogris, E., Du, X., Nelson, K.C., Mak, E.K., Yu, X.X., Lane, W.S., and Pallas, D.C. 1999a. A protein phosphatase methylesterase (PME-1) is one of several novel proteins stably associating with two inactive mutants of protein phosphatase $2 \mathrm{~A}$. J. Biol.Chem. 274: 14382-14391.

Ogris, E., Mudrak, I., Mak, E., Gibson, D., and Pallas, D.C. 1999b. Catalytically inactive protein phosphatase 2A can bind to polyomavirus middle tumor antigen and support complex formation with pp60 ${ }^{\text {c-src }}$. J. Virol. 73: 7390-7398.

Pear, W.S., Nolan, G.P., Scott, M.L., and Baltimore, D. 1993. Production of high-titer helper-free retroviruses by transient transfection. Proc. Natl. Acad. Sci.90: 8392-8396.

Rempola, B., Kaniak, A., Migdalski, A., Rytka, J., Slonimski, P.P., and di Rago, J.P. 2000. Functional analysis of RRD1 (YIL153w) and RRD2 (YPL152w), which encode two putative activators of the phosphotyrosyl phosphatase activity of PP2A in Saccharomyces cerevisiae. Mol. Gen. Genet. 262: 1081-1092.

Ronne, H., Carlberg, M., Hu, G.Z., and Nehlin, J.O. 1991. Protein phosphatase 2A in Saccharomyces cerevisiae: Effects on cell growth and bud morphogenesis. Mol. Cell. Biol. 11: 4876-4884.

Sneddon, A.A., Cohen, P.T., and Stark, M.J. 1990. Saccharomyces cerevisiae protein phosphatase $2 \mathrm{~A}$ performs an essential cellular function and is encoded by two genes. $E M B O$ J. 9: 4339-4346.

Sola, M.M., Langan, T., and Cohen, P. 1991. p34cdc2 phosphorylation sites in histone $\mathrm{H} 1$ are dephosphorylated by protein phosphatase $2 \mathrm{~A}_{1}$. Biochim. Biophys. Acta 1094: 211216.

Sutton, A., Immanuel, D., and Arndt, K.T. 1991. The SIT4 protein phosphatase functions in late $\mathrm{G} 1$ for progression into $\mathrm{S}$ phase. Mol. Cell. Biol. 11: 2133-2148.

Van Hoof, C., Cayla, X., Bosch, M., Merlevede, W., and Goris, J. 1994. The phosphotyrosyl phosphatase activator of protein phosphatase 2A. A novel purification method, immunologi- 
Fellner et al.

cal and enzymic characterization. Eur. J. Biochem. 226: 899907.

Van Hoof, C., Janssens, V., De Baere, I., Stark, M.J., de Winde, J.H., Winderickx, J., Thevelein, J.M., Merlevede, W., and Goris, J. 2001. The Saccharomyces cerevisiae phosphotyrosyl phosphatase activator proteins are required for a subset of the functions disrupted by protein phosphatase $2 \mathrm{~A}$ mutations. Exp. Cell Res. 264: 372-387.

Virshup, D.M. 2000. Protein phosphatase 2A: A panoply of enzymes. Curr. Opin. Cell Biol. 12: 180-185.

Wach, A., Brachat, A., Alberti-Segui, C., Rebischung, C., and Philippsen, P. 1997. Heterologous HIS3 marker and GFP reporter modules for PCR-targeting in Saccharomyces cerevisiae. Yeast 13: 1065-1075.

Wei, H., Ashby, D.G., Moreno, C.S., Ogris, E., Yeong, F.M., Corbett, A.H., and Pallas, D.C. 2001. Carboxymethylation of the PP2A catalytic subunit in Saccharomyces cerevisiae is required for efficient interaction with the B-type subunits Cdc55p and Rts1p. J. Biol. Chem. 276: 1570-1577.

Wu, J., Tolstykh, T., Lee, J., Boyd, K., Stock, J.B., and Broach, J.R. 2000. Carboxyl methylation of the phosphoprotein phosphatase $2 \mathrm{~A}$ catalytic subunit promotes its functional association with regulatory subunits in vivo. EMBO J. 19: 5672-5681.

Zolnierowicz, S. 2000. Type 2A protein phosphatase, the complex regulator of numerous signaling pathways. Biochem. Pharmacol. 60: 1225-1235. 


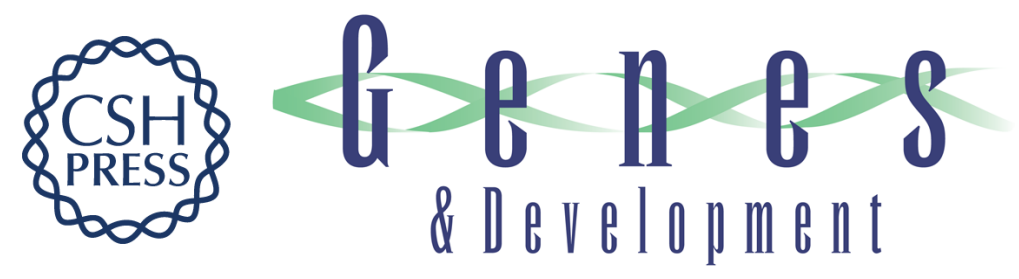

\section{A novel and essential mechanism determining specificity and activity of protein phosphatase 2A (PP2A) in vivo}

Thomas Fellner, Daniel H. Lackner, Hans Hombauer, et al.

Genes Dev. 2003, 17:

Access the most recent version at doi:10.1101/gad.259903

References This article cites 38 articles, 17 of which can be accessed free at: http://genesdev.cshlp.org/content/17/17/2138.full.html\#ref-list-1

License

Email Alerting

Receive free email alerts when new articles cite this article - sign up in the box at the top Service right corner of the article or click here.

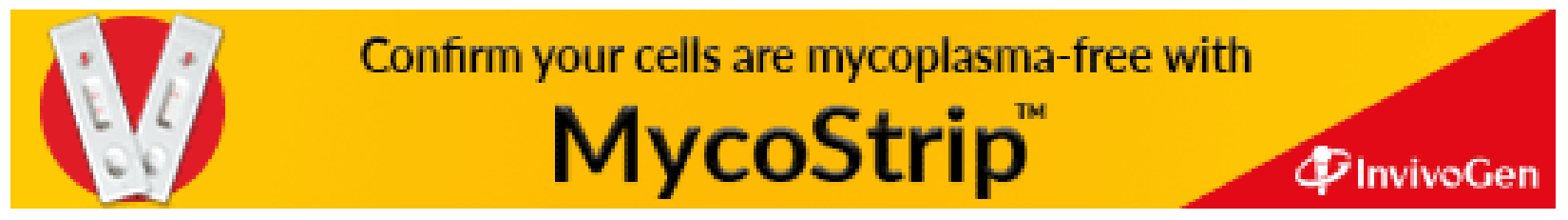

